\title{
Cyclooxygenase-2 Expression in Invasive Breast Carcinomas of No Special Type and Correlation with Pathological Profiles Suggest a Role in Tumorigenesis Rather than Cancer Progression
}

\author{
Nurul Akmar Misron ${ }^{1,2 *}$, Lai-Meng Looi ${ }^{1}$, Nik Raihan Nik Mustapha ${ }^{2}$
}

\begin{abstract}
Background: COX-2 has been shown to play an important role in the development of breast cancer and increased expression has been mooted as a poor prognostic factor. The purpose of this study was to investigate the relationship between COX-2 immunohistochemical expression and known predictive and prognostic factors in breast cancer in a routine diagnostic histopathology setting. Materials and Methods: Formalin-fixed paraffinembedded tumour tissue of 144 no special type (NST) invasive breast carcinomas histologically diagnosed between January 2009 and December 2012 in Hospital Sultanah Bahiyah, Alor Setar, Kedah were immunostained with COX-2 antibody. COX-2 overexpression was analysed against demographic data, hormone receptor status, HER2neu overexpression, histological grade, tumour size and lymph node status. Results: COX-2 was overexpressed in 108/144 (75\%) tumours and was significantly more prevalent $(87 \%)$ in hormone receptor-positive tumours. There was no correlation between COX-2 overexpression and HER2/neu status. Triple negative cancers had the lowest prevalence $(46 \%) \quad(p<0.05)$. A rising trend of COX-2 overexpression with increasing age was observed. There was a significant inverse relationship with tumour grade $(p<0.05)$, prevalences being $94 \%, 83 \%$ and $66 \%$ in grades 1, 2 and 3 tumours, respectively. A higher prevalence of COX-2 overexpression in smaller size tumours was observed but this did not reach statistical significance. There was no relationship between COX-2 expression and lymph node status. Conclusions: This study did not support the generally held notion that COX-2 overexpression is linked to poor prognosis, rather supporting a role in tumorigenesis. Larger scale studies with outcome data and basic studies on cancer pathogenetic pathways will be required to cast further light on whether COX-2 inhibitors would have clinical utility in cancer prevention or blockage of cancer progression. In either setting, the pathological assessment for COX-2 overexpression in breast cancers would have an important role in the selection of cancer patients for personalized therapy with COX-2 inhibitors.
\end{abstract}

Keywords: Cyclooxygenase-2 (COX-2) - NOS-type invasive ductal breast carcinoma - immunohistochemistry.

Asian Pac J Cancer Prev, 16 (4), 1553-1558

\section{Introduction}

Invasive breast carcinoma is the most common cancer in women worldwide, with an incidence in developed countries $(71.7 / 100,000)$ that is considerably higher than lower income countries $(29.3 / 100,000)$ (Youlden et al., 2012). In Malaysia, a rapidly developing country, 3,242 new female breast cancers were reported to the Malaysian cancer registry in 2007, making it the most commonly diagnosed cancer in Malaysian women. It accounted for $18.1 \%$ of all cancers registered and $32.1 \%$ of all female cancers with a peak incidence at the 50-59 years age-group. The incidence of breast cancer was highest among Chinese females where the age standardized rate (ASR) was 38.1 per 100,000 population, followed by ethnic Indians and Malays with ASRs of 33.7 per 100,000 population and 25.4 per 100,000 population respectively (Zainal Ariffin et al., 2011).

The aetiology of breast cancer is multifactorial, implicating reproductive factors, hormonal imbalances, genetic predispositions and more recently, diet and metabolic factors (McPherson et al., 2000; Azrad and Demark-Wahnefried, 2014; Dean et al., 2014). Breast carcinoma has heterogenous histomorphology (Sinn and Kreipe, 2013). The WHO class of invasive carcinoma of no special type (NST) forms the largest proportion of breast carcinomas, comprising between $40 \%$ and $75 \%$ in published literature. Other types of invasive breast carcinoma include lobular, tubular, cribriform, medullary, mucin-producing, papillary, metaplastic, secretory, adenoid and other rarer types and variants (Lakhani et al., 2012). 
Over the years, there has been crucial development in the molecular profiling of invasive breast carcinoma for targeted therapy (Yersal and Barutca, 2014). Immunohistochemical assessment based on hormone (oestrogen and progesterone) receptor expression and HER2/neu oncoprotein overexpression is now widely used to delineate surrogate luminal (hormone positive), HER2 and basal-like/triple negative immunophenotypes (Schnitt, 2010). There is ample evidence that the presence of oestrogen and/or progesterone hormone receptors in the tumour correlates well with response to hormonal therapy. In contrast, the overexpression of HER2/neu oncoprotein is associated with poor prognosis particularly when lymph node metastases are present but confers response to targeted therapy with Herceptin ${ }^{\circledR}$ (trastuzumab) (Schnitt, 2010; Yersal and Barutca, 2014). In the evaluation of breast carcinoma, oestrogen and progesterone receptor status and HER2/neu overexpression are the most accepted predictive factors, while histological grade, tumour size and lymph node status are the most evidence-based pathological prognostic factors.

Notwithstanding, there remains considerable interest in additional prognostic and predictive factors which may help to further fine-tune the therapy of breast cancer in today's climate of personalized medicine. Many studies have shown that cyclooxygenase-2 (COX-2) plays an important role in the development of some human cancers particularly pulmonary, colon and breast carcinoma and its preinvasive lesion, ductal carcinoma-in-situ (DCIS) (Soslow et al., 2000; Perrone et al., 2005; Ciris et al., 2011; Markkula1 et al., 2014). Cyclooxygenase (COX) is the rate-limiting enzyme in the biosynthesis of eicosanoids from arachidonic acid. There are $2 \mathrm{COX}$ isoforms: the constitutive form, $\mathrm{COX}-1$, is involved in processes such as parturition and platelet aggregation; the inducible form, COX-2, is involved in inflammatory reactions as well as ovulation, implantation, perinatal renal development and remodeling of the ductus arteriosus. COX-2 expression is induced by a variety of proinflammatory agents, growth factors, tumour promoters and mitogens. COX-2 has been shown to stimulate proliferation, angiogenesis, invasiveness, inhibit apoptosis and predispose the mammary gland to carcinogenesis (Furstenberger et al., 2006; De Moraes et al., 2007; Lu et al., 2007; Markkula1 et al., 2014).

COX-2 expression has been shown to correlate with aromatase expression within human breast cancer tissue (Lee et al., 2010). Oestrogens are produced from androgens by the action of the aromatase enzyme. In postmenopausal women, plasma oestrogens result from peripheral aromatization, particularly in adipose tissue. Many breast cancers, however, also contain aromatase and synthesize oestrogens by intratumoral aromatase activity. The COX-2 products PGE 2 and cytokines such as interleukin-6 (IL-6) or TNF- $\alpha$ have the ability to stimulate aromatase activity and subsequently contribute to cancer progression particularly in hormone-positive breast cancer (Brodie, 2001; Baumgarten and Frasor, 2012; Simpson and Brown, 2013).

COX-2 overexpression in breast cancer provides an attractive basis for the use of COX-2 inhibitors in the prevention and treatment of breast cancer (Ristimaki et al., 2002; Wulfing et al., 2003; Witton et al., 2004; Nam et al., 2005; Cho et al., 2006; Park et al., 2006; Schmitz et al., 2006; Dalamaga, 2013 ). Some studies have indicated that COX-2 inhibitors can contribute to increased survival among women with breast cancer (Holmes et al., 2010) or have a protective effect against breast cancer (Sharpe et al., 2000).

We carried out a study for COX-2 immunohistochemical expression in archival breast carcinoma material to better appreciate its pattern of expression and correlation with routine predictive and prognostic parameters within the diagnostic histopathology laboratory setting, as well as its pathophysiological implications.

\section{Materials and Methods}

This is a retrospective and observational study. Archival paraffin blocks of breast carcinomas (biopsies or mastectomies) from January 2009 to December 2010, classified as WHO invasive breast carcinoma of no special type (NST) (Lakhani et al., 2012), were identified from the records of the pathology department, hospital Sultanah Bahiyah, Kedah, Malaysia. Each case had to have at least one formalin-fixed, paraffin-embedded tissue block containing NST invasive breast carcinoma to qualify for the study. NST breast carcinoma with unavailable tissue blocks or suboptimal tissue (crushed, chemotherapy effect) and all other special histological types of breast cancers were excluded from the study.

The patients' demographic data were obtained from the histopathology request form. Each patient was identified through a research code so that the identities of the patients were anonymised. The study was approved by the institutional medical ethics committee.

\section{Evaluation for predictive and prognostic factors}

All the predictive (oestrogen and progesterone receptor status and HER2/neu expression) and prognostic (histological grade-modified, tumour size, lymphovascular invasion and lymph node status) parameters were obtained from the histopathological report. However, some of the cases, especially biopsies, did not have complete information about the prognostic parameters.

Expression for oestrogen and progesterone receptors and HER2/neuproteins were assessed using standard immunohistochemical protocols. Oestrogen and progesterone receptor status were considered positive when at least $1 \%$ of tumour cells show nuclear staining (Hammond et al., 2010). HER2/neu was considered overexpressed when at least $30 \%$ of tumour cells exhibit strong complete membrane staining (Wolff et al., 2007).

\section{Immunohistochemistry for $\mathrm{COX}-2$ expression}

Immunohistochemical staining was performed using COX-2 antibody (LabVision-RM-9121-S Clone SP21 Rabbit monoclonal) at 1:50 dilution according to standard immunohistochemical staining protocols. Aliver cirrhosis sample with strong hepatocyte staining intensity was used as positive control.

The stained sections were then examined under light 
COX-2 Expression in Invasive Breast Carcinomas Suggests a Role in Tumorigenesis Rather than Cancer Progression

microscopy against negative and positive control slides. Cytoplasmic immunoreactivity of COX-2 was given an immunohistochemical score (HIS), based on the Germany ImmunoReactive Score (Soslow et al., 2000). This score was calculated by combining an estimate of the percentage of immunoreactive tumour cells (quantity score) with an estimate of the staining intensity (staining intensity score), as follows: no staining was scored as $0,1-10 \%$ of cells stained scored as $1,11-50 \%$ as $2,51-80 \%$ as 3 and $81-100 \%$ as 4 . Staining intensity was rated on a scale of 0 to 3 , with $0=$ negative, $1=$ weak, $2=$ moderate and $3=$ strong (Figure 1). The raw data were converted to the HIS by multiplying the quantity and staining intensity scores. Theoretically, the score could range from 0 to 12. An HIS score of 9-12 was considered strong immunoreactivity, 5-8 was considered moderate, 1-4 was considered weak and 0 was scored as negative. COX-2 is considered overexpressed if the HIS score was moderate to strong.

The immunostained slides were reviewed by two evaluators independently and a consensus was reached through re-evaluation of cases that show discrepancies in their scores.

\section{Statistical analysis}

Statistical analysis was performed using the chi-square test (SPSS, version 17.0) with statistical significance set at $\mathrm{p}<0.05$.

\section{Results}

A total of 144 histologically confirmed invasive breast carcinomas of no special type were included in this study: 70 were biopsies and the rest were mastectomies. The ages of patients ranged from 26 years to 82 years ( mean $=50$ years; median $=51$ years). Only one patient was male $(0.7 \%)$. Ethnically, there were 104 Malays $(72.2 \%)$, 27 Chinese (18.8\%), 11 Indians (7.6\%) and 2 of other ethnicity $(1.4 \%)$.

\section{COX-2 expression}

COX-2 overexpression was detected in $108(75 \%)$ of the cancers studied (Table 1). COX-2 overexpression was significantly more prevalent $(87 \%)$ in hormone receptor-positive (ER/PR positive, HER2/neu negative and ER/PR positive, HER2/neu overexpressed) tumours compared with hormone receptor negative tumours (COX2 overexpression prevalence of $59 \%)(\mathrm{p}<0.05)$ There was no correlation between $\mathrm{COX}-2$ overexpression and HER2/neu negative status (ER/PR positive, HER2/neu negative and triple negative), the prevalence being $73 \%$. Triple negative cancers had the lowest prevalence (46\%) of COX-2 overexpression $(\mathrm{p}<0.05)$.

Table 2 summarises the comparison of COX2 expression with age and pathological prognostic factors. Although ethnic Malays constituted $72 \%$ of the study population, Malays contributed to only $51 \%$ of cancers with COX-2 overexpression. $55 \%$ of COX-2 overexpressed cancers occurred between the ages of 3554 years, which was also the largest age-group (56\%) in this study. Nevertheless, there appears to be a rising trend of COX-2 overexpression with increasing age (Table

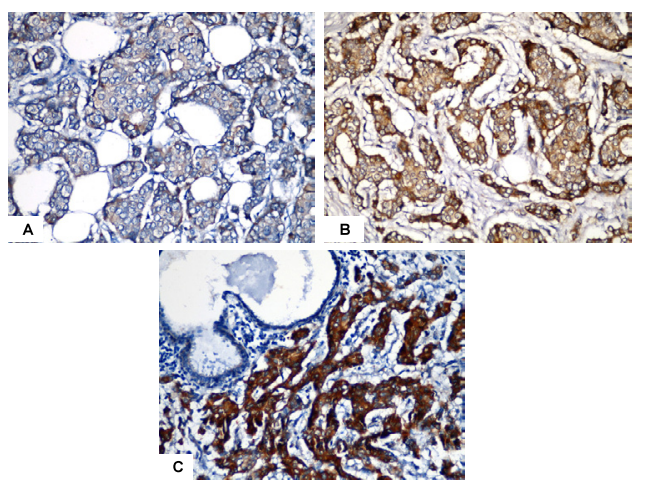

Figure 1. COX-2 Staining Intensity.A. Weak (Score 1). B. Moderate (Score 2). C. Strong (Score 3)

Table 1. Expression of COX-2 Against Predictive Parameters in NOS-type Invasive Ductal Breast Carcinomas.

\begin{tabular}{|c|c|c|}
\hline $\begin{array}{l}\text { Hormone receptor/ } \\
\text { HER2 status }\end{array}$ & $\begin{array}{c}\text { COX-2 } \\
\text { Overexpressed }\end{array}$ & $\begin{array}{l}\text { COX-2 Not } \\
\text { overexpressec }\end{array}$ \\
\hline \multicolumn{3}{|c|}{ ER/PR positive, HER $2 /$ neu negative } \\
\hline & $49(86.0 \%)$ & $8(14.0 \%)$ \\
\hline \multicolumn{3}{|c|}{ ER/PR positive, HER $2 /$ neu overexpressed } \\
\hline & $23(88.5 \%)$ & $3(11.5 \%)$ \\
\hline \multicolumn{3}{|c|}{ ER/PR negative, HER2/neu overexpressed } \\
\hline & $23(69.7 \%)$ & $10(3$ \\
\hline Triple 1 & $13(46.4 \%)$ & $15(53.6 \%)$ \\
\hline Total & $108(75 \%)$ & $36(25 \%)$ \\
\hline
\end{tabular}

Table 2. COX-2 Expression in Relation to Clinicopathological Parameters.

\begin{tabular}{|c|c|c|c|c|}
\hline $\begin{array}{r}\text { Clinicopatholo } \\
\text { parameter }\end{array}$ & gical & Overexpressed & $\begin{array}{c}\text { COX-2 } \\
\text { Not }\end{array}$ & $\mathrm{P}$ value \\
\hline Age (years) & $\mathrm{n}$ & & & \\
\hline$<35$ & 10 & $7(70.0 \%)$ & $3(30.0 \%)$ & \\
\hline $35-54$ & 80 & $59(73.8 \%)$ & $21(26.2 \%)$ & - \\
\hline$>55$ & 54 & $42(77.8 \%)$ & $12(22.2 \%)$ & \\
\hline Tumour size & & & & \\
\hline $2 \mathrm{~cm}$ or less & 11 & $10(90.9 \%)$ & $1(9.1 \%)$ & \\
\hline$>2$ to $5 \mathrm{~cm}$ & 41 & $32(78.0 \%)$ & $9(22.0 \%)$ & 0.23 \\
\hline$>5 \mathrm{~cm}$ & 33 & $22(66.7 \%)$ & $11(33.3 \%)$ & \\
\hline NA & 59 & & & \\
\hline Tumour grade* & & & & \\
\hline 1 & 17 & $16(94.1 \%)$ & $1(5.9 \%)$ & \\
\hline 2 & 48 & $40(83.3 \%)$ & $8(16.7 \%)$ & 0.02 \\
\hline 3 & 65 & $43(66.2 \%)$ & $22(33.8 \%)$ & \\
\hline NA & 14 & & & \\
\hline Lymphovascula & ar inv & sion & & \\
\hline Yes & 40 & $27(67.5 \%)$ & $13(32.5 \%)$ & 0.17 \\
\hline No & 46 & $37(80.4 \%)$ & $9(19.6 \%)$ & \\
\hline NA & 58 & & & \\
\hline Lymph node in & vasio & & & \\
\hline Yes & 55 & $40(72.7 \%)$ & $15(27.3 \%)$ & 0.62 \\
\hline No & 18 & $12(66.7 \%)$ & $6(33.3 \%)$ & \\
\hline NA & 71 & & & \\
\hline
\end{tabular}

*NA: Not available. This applies to biopsies where these parameters could not be reliably assessed.

2). There was a significant inverse relationship between COX-2 overexpression and tumour grade $(\mathrm{p}<0.05)$, in that there was a higher prevalence of COX-2 overexpression in low grade tumours.

There was a higher prevalence of COX-2 overexpression 
in smaller size tumours and tumours with no evidence of lymphovascular invasion. However, overexpression was seen more in tumours with lymph node invasion. Notwithstanding, these findings did not reach statistical significance.

\section{Discussion}

The age and ethnic distribution for breast carcinomas in this study differs from nationwide data as the demographic distribution in Kedah is different and does not represent the general population in Malaysia (Zainal Ariffin et al., 2011). Furthermore, all the cases were from hospital Sultanah Bahiyah and did not include cases from other hospitals in Kedah. Due to the small number of non-Malay patients in this study, it is not possible to draw conclusions on correlations with ethnicity.

In this study, COX-2 overexpression was seen in $75 \%$ of the cancers studied. The reported prevalence of COX-2 expression in breast cancer has varied from $4.5 \%$ to $85 \%$ (Ristimaki et al., 2002; Glover et al., 2011; Markkula1 et al., 2014). This wide range is likely to be due to unclear and inconsistent definition of increased COX-2 expression in many studies and the different evaluation systems used (Lee et al., 2010). We have used a defined scoring system with consensus agreement between two independent evaluators. Notably, our findings are within the reported range of other workers.

Our study revealed a higher prevalence of COX-2 overexpression in hormone-receptor positive cancers as well as in lower histological grades and small-size tumours. A lower prevalence was noted in triple negative cancers. We did not find an association between COX-2 overexpression and lymph node status or HER2/Neu overexpression. This appears to be in contradiction to the concept that COX-2 expression connotes a poor prognosis. A similar observation with regards hormone receptor status was reported recently by researchers from Norway, who also noted that COX-2 expression did not connote a poorer breast cancer-specific survival (Dhakal et al., 2012). In view of this, the general view that COX2 expression is a poor prognostic marker should be reexamined. The interaction of COX-2 with oestrogen is a complex and controversial one, particularly with regards to cause and effect. COX-2 activation of aromatase through prostaglandin production, leading to conversion of androgen to oestrogen in breast tissues and activation of the oestrogen receptor and its target genes, has been mooted as the likely pathway whereby neoplastic cell growth is stimulated (Ciris et al., 2011). Recent studies have also highlighted the role of the COX-2-prostaglandin E2-aromatase pathway in obesity and the proliferation of oestrogen-positive cancers (Baumgarten and Frasor, 2012; Simpson and Brown, 2013). In view of that, COX2 expression may contribute more to tumorigenesis than to progression of already invasive cancers. Such a notion would be in keeping with COX-2 expression in carcinoma-in-situ and early cancers, which have been reported in various neoplastic systems (Glover et al., 2011). It would be compatible with our findings of its expression in hormone-positive, smaller size and lower grade cancers. It would also be consistent with growing evidence that COX-2 inhibitors have a role in cancer prevention (Harris, 2009).

A rising prevalence of COX-2 expression with increasing age was also observed in our study. A higher prevalence of hormone-receptor positivity is also wellknown in breast cancers occurring in postmenopausal women (Tarone and Chu, 2002). In view of the interplay between COX-2 and oestrogen, the above observation may be a reflection of hormone receptor status rather than age.

Notwithstanding the above arguments, it cannot be ignored that many studies have observed that COX-2 was overexpressed in hormone receptor-negative and HER2/ neu overexpressed breast carcinomas (Ristimaki et al., 2002; Wulfing, et al., 2003; Nam et al., 2005; Mohammad et al., 2006; Miglietta et al., 2010) and have linked it to poorer prognosis. We have not studied whether COX-2 expression is linked to the more aggressive oestrogenpositive cancers as outcome data was not available. We also have not evaluated our findings against obesity, breast size and BMI data and recognize that this may useful in view of the metabolic pathways of COX-2. This is also pertinent in view of the rising incidence of both obesity and breast cancer among Malaysian women. Larger scale studies with social and outcome data and basic studies on cancer pathogenetic pathways will be required to cast further light on whether COX-2 is truly a poor prognostic factor or largely a cancer promoting agent.

COX-2 inhibitors are relatively cheap and affordable compared to other drugs used in the treatment of breast cancer such as tamoxifen and Herceptin. They are also relatively tolerable with minimal severe side effects. Their clinical utility in either cancer prevention or blockage of cancer progression is worthy of consideration (Holmes, 2010). In either setting, the pathological assessment for COX-2 overexpression in breast cancers would have an important role in the selection of cancer patients for personalized therapy with COX-2 inhibitors.

Interestingly, recent studies into polymorphisms of the COX-2 gene have not shown conclusive links with COX-2 expression in breast cancer suggesting that the molecular pathway of COX-2 activation is a complex one (Markkula1 et al., 2014). The possibility that COX2 expression may involve associations with other genes such as MMP-2, as has been observed in colorectal cancer (Shalaby et al., 2014), is worthy of further investigation.

\section{Acknowledgements}

This study was supported by an FRGS IPTA (KPT1053/2011) grant on "Enhancing personalized medicine through pathological profiling of cancer" awarded to Professor LM Looi and carried out as a research project in partial fulfillment of a Master of Pathology degree of the first author. The authors declare no conflict of interest in the conduct of this study. We also would like to thank the Director-General of Health of Malaysia for his support of this project and permission to publish the findings. 


\section{References}

Azrad M, Demark-Wahnefried W (2014). The association between adiposity and breast cancer recurrence and survival: A review of the recent literature. Curr Nutr Rep, 1, 9-15.

Baumgarten SC, Frasor J (2012). Minireview: inflammation: an instigator of more aggressive estrogen receptor $(E R)$ positive breast cancers. Mol Endocrinol, 26, 360-71.

Brodie AM, Lu Q, Long BJ, et al (2001). Aromatase and COX-2 expression in human breast cancers. J Steroid Biochem Mol Biol, 79, 41-7

Cho MH, Yoon JH, Jaegal YJ, et al (2006). Expression of cyclooxygenase- 2 in breast carcinogenesis and its relation to HER-2/neu and $\mathrm{p} 53$ protein expression in invasive ductal carcinoma. Breast, 15, 390-8.

Ciris IM, Bozkurt KK, Baspınar S, Kapucuoglu FN (2011). Immunohistochemical Cox-2 overexpression correlates with Her-2/Neu overexpression in invasive breast carcinomas: a pilot study. Pathol Res Pract, 207, 182-7

Dhakal HP, Naume B, Synnestvedt M, et al (2012). Expression of cyclooxygenase- 2 in invasive breast carcinomas and its prognostic impact. Histol Histopathol, 27, 1315-25.

Dalamaga M (2013). Obesity, insulin resistance, adipocytokines and breast cancer: new biomarkers and attractive therapeutic targets. World J Exp Med, 3, 34-42

De Moraes E, Dar NA, de Moura Gallo CV, Hainaut P (2007). Cross-talks between cyclooxygenase-2 and tumor suppressor protein p53: Balancing life and death during inflammatory stress and carcinogenesis. Int J Cancer, 121, 929-37.

Dean SJ, Perks CM, Holly JM, et al (2014). Loss of PTEN expression is associated with IGFBP2 expression, younger age and late stage in triple-negative breast cancer. Am J Clin Pathol, 141, 323-33

Nam E, Lee SN, Im SA, et al (2005). Expression of cyclooxygenase-2 in human breast cancer: relationship with HER-2/neu and other clinicopathological prognostic factors. Cancer Res Treat, 37, 165-70.

Furstenberger G, Krieg P, Muller-Decker K, Habenicht AJ (2006). What are cyclooxygenases and lipoxygenases doing in the driver's seat of carcinogenesis? Int J Cancer, 119, 2247-54.

Glover JA, Hughes CM, Cantwell MM, Murray LJ (2011). A systematic review to establish the frequency of cyclooxygenase- 2 expression in normal breast epithelium, ductal carcinoma in situ, microinvasive carcinoma of the breast and invasive breast cancer. Br J Cancer, 105, 13-7.

Hammond MEH, Hayes DF, Dowsett M, et al (2010). American society of clinical oncology/college of American pathologists guideline recommendations for immunohistochemical testing of estrogen and progesterone receptors in breast cancer. Arch Pathol Lab Med, 134, 907-22.

Harris RE (2009). Cyclooxygenase-2 (COX-2) blockade in the chemoprevention of cancers of the colon, breast, prostate and lung. Inflammopharmacology, 17, 55-67.

Holmes MD, Chen WY, Li L, et al (2010). Aspirin intake and survival after breast cancer. J Clin Oncol, 28, 1467-72.

Lakhani S, Ellis I, Schnitt S, et al (2012). WHO Classification of Tumours of the Breast, 4th ed. Lyon, IARC Press.

Lee JA, Bae JW, Woo SU, Kim H, Kim CH (2010). Correlation between COX-2 expression and hormone receptors in invasive ductal breast cancer. J Korean Surg Soc. 78, 140-8

Lu S, Yu G, Zhu Y, Archer MC (2005). Cyclooxygenase-2 overexpression in MCF-10F human breast epithelial cells inhibits proliferation, apoptosis and differentiation and causes partial transformation. Int J Cancer, 116, 847-52.

McPherson K, Steel CM, Dixon JM (2000). ABC of breast diseases. Breast cancer-epidemiology, risk factors and genetics. $B M J$, 321, 624-8

Markkula1 A, Simonsson M, Rosendahl AH (2014). Impact of COX2 genotype, ER status and body constitution on risk of early events in different treatment groups of breast cancer patients. Int J Cancer, 135, 1898-910.

Miglietta A, Toselli M, Ravarino N, et al (2010). COX-2 expression in human breast carcinomas: correlation with clinicopathological features and prognostic molecular markers. Expert Opin Ther Targets, 14, 655-64.

Mohammad AM, Abdel HA, Abdel W, et al (2006). Expression of cyclooxygenase-2 and 12-lipoxygenase in human breast cancer and their relationship with HER-2/neu and hormonal receptors: impact on prognosis and therapy. Indian J Cancer, 43,163-8.

Park K, Han S, Shin E, Kim HJ, Kim JY (2006). Cox-2 expression on tissue microarray of breast cancer. Eur J Surg Oncol, 32, 1093-6

Perrone G, Santini D, Vincenzi B, et al (2005). COX-2 expression in DCIS: correlation with VEGF, HER-2/neu, prognostic molecular markers and clinicopathological features. Histopathology, 46, 561-8.

Ristimäki A, Sivula A, Lundin J, et al (2002). Prognostic significance of elevated cyclooxygenase- 2 expression in breast cancer. Cancer Res, 62, 632-5.

Schmitz KJ, Callies R, Wohlschlaeger J, et al (2006). Overexpression of cyclo-oxygenase- 2 is an independent predictor of unfavourable outcome in node-negative breast cancer, but is not associated with protein kinase B (Akt) and mitogen-activated protein kinase (ERK1/2, p38) activation or with Her-2/neu signalling pathways. J Clin Pathol, 59, 685-91.

Schnitt SJ (2010). Classification and prognosis of invasive breast cancer: from morphology to molecular taxonomy. Modern Pathology, 23, 60-4

Shalaby MA, Nounou HA, Alanazi MS, et al (2014). Associations between single nucleotide polymorphisms of COX-2 and MMP-2 genes and colorectal cancer susceptibility in the Saudi population. Asian Pac J Cancer Prev, 15, 4989-94.

Sinn H-P, Kreipe H (2013). A brief overview of the who classification of breast tumors, 4th edition, focusing on issues and updates from the 3 rd edition. Breast Care, 8, 149-54.

Sharpe CR, Collet JP, McNutt M, et al (2000). Nested case-control study of the effects of non-steroidal antiinflammatory drugs on breast cancer risk and stage. $\mathrm{Br} J$ Cancer, 83, 112-20.

Simpson ER, Brown KA (2013). Obesity and breast cancer: role of inflammation and aromatase. J Mol Endocrinol, 51, 51-9.

Soslow RA, Dannenberg AJ, Rush D, et al (2000). COX-2 is expressed in human pulmonary, colonic and mammary tumors. Cancer, 89, 2637-45.

Tarone RE, Chu KC (2002). The greater impact of menopause on ER-than ER+breast cancer incidence: a possible explanation (United States). Cancer Causes Control, 13, 7-14.

Witton CJ, Hawe SJ, Cooke TG, Bartlett JM (2004). Cyclooxygenase 2 (COX2) expression is associated with poor outcome in ER-negative, but not ER-positive, breast cancer. Histopathology, 45, 47-54.

Wolff AC, Hammond ME, Schwartz JN, et al (2007). American Society of clinical oncology/college of American pathologists guideline recommendations for human epidermal growth factor receptor 2 testing in breast cancer. Arch Pathol Lab Med, 131, 18-43.

Wulfing P, Diallo R, Muller C, et al (2003). Analysis of cyclooxygenase- 2 expression in human breast cancer: high throughput tissue microarray analysis. J Cancer Res Clin Oncol, 129, 375-82.

Yersal O, Barutca S (2014). Biological subtypes of breast 
Nurul Akmar Misron et al

cancer: prognostic and therapeutic implications. World $J$ Clin Oncol, 5, 412-24

Youlden DR, Cramb SM, Dunn NA, et al (2012). The descriptive epidemiology of female breast cancer: an international comparison of screening, incidence, survival and mortality. Cancer Epidemiol, 36, 237-48.

Zainal Ariffin O, Nor Saleha IT (2011). National cancer registry report 2007. In O Zainal Ariffin and I. T. N. Saleha (Eds.). Kuala Lumpur: Ministry of Health. 